\title{
Influence of frozen storage on the fatty acid composition of ostrich meat enriched with linseed and rapeseed
}

\author{
J.O. Horbańczuk ${ }^{1}$, E. Poławska ${ }^{1}$, A. Wójcik ${ }^{2}$ \& L.C. Hoffman ${ }^{3 \#}$ \\ ${ }^{1}$ Polish Academy of Sciences, Institute of Genetics and Animal Breeding, 05-552, Jastrzębiec, Poland \\ 2 Department of Animal Hygiene and Environmental, Faculty of Animal Bioengineering, University of Warmia and Mazury, \\ 10-719 Olsztyn, Poland \\ ${ }^{3}$ Department of Animal Sciences, University of Stellenbosch, Private Bag X1, Matieland, 7602, South Africa
}

(Received 25 July 2014; Accepted 2 April 2015; First published online 16 May 2015)

\author{
Copyright resides with the authors in terms of the Creative Commons Attribution 2.5 South African Licence. \\ See: http://creativecommons.org/licenses/by/2.5/za \\ Condition of use: The user may copy, distribute, transmit and adapt the work, but must recognise the authors and the South \\ African Journal of Animal Science.
}

\begin{abstract}
The aim of the study was to evaluate the effect of the duration (24 hours, 60 days and 120 days) of frozen storage $\left(-20^{\circ} \mathrm{C}\right)$ on the fatty acid composition of meat from ostriches supplemented with linseed and rapeseed. The study was carried out on muscles of 40 ostriches raised on five dietary groups: control with no supplementation (C), with 4\% linseed (L4); 8\% linseed (L8); and 5\% rapeseed (R5); or 10\% rapeseed (R10) in the diet. As the frozen storage period increased, the fatty acid profile of the ostrich meat in all the "enriched" groups changed, especially treatments L4 and L8. There was a decrease in the polyunsaturated fatty acid content (especially from 61 to 120 days of storage) including linolenic, arachidonic and docosahexaenoic acids. However, storage did not influence the fatty acid profile of ostrich meat up to 60 days. These results suggest that freezing is an acceptable method for preserving ostrich meat (up to 60 days), causing only a small decrease in the fatty acids of ostrich meat enriched with n-3 fatty acids. However, further research on prolonged frozen storage is recommended.
\end{abstract}

Keywords: Exotic meat; frozen, lipids; n-3; n-6; poultry, diet, Struthio camelus

\# Corresponding author: Ich@sun.ac.za

\section{Introduction}

In recent years, consumers have paid particular attention to the quality and health effects of foods. Nowadays they are becoming increasingly aware that food with a high n-3 polyunsaturated fatty acids (PUFA) content and well-balanced polyunsaturated fatty acids/saturated fatty acids (PUFA/SFA) are important for human health for the role that they play in protection against cardiovascular and inflammatory diseases (Dalle Zotte et al., 2013). According to the World Health Organization (WHO) recommendations, n-6/n-3 and PUFA/SFA ratios should be below 4 and above 0.4 , respectively (WHO/FAO, 2003). Increased interest in enhancing the nutritional quality of meat foods has stimulated research on manipulating their fatty acid composition through nutritional strategies (Hoffman et al., 2005; Diaz et al., 2011; Polawska et al., 2013), including dietary supplementation with n-3 fatty acid-enriched sources such as fish, linseed and rapeseed oils. Ostrich meat is gaining in popularity among modern consumers (Horbanczuk et al., 1998, 2008; Sales \& Horbanczuk 1998; Cooper \& Horbanczuk 2004). When enriched with oil seeds, it has been shown to have beneficial PUFA/SFA (0.4 - 0.7) and n-6/n-3 ratios (2 - 4) (Poławska et al., 2013). In addition, the level of inclusion of oil seeds in the diet influences the level of fatty acid deposition (Hoffman et al., 2005; Poławska et al., 2012; 2013) and it is therefore worthwhile to evaluate a range of oil seed inclusion levels.

Ostrich meat is typically sold as entire muscles (Sales \& Horbańczuk, 1998; Hoffman, 2008). Although a large number of these muscles are sold as "fillets" or "steaks", they differ in fibre types as well as chemical composition (Hoffman et al., 2005; Girolami et al., 2003]). It is therefore important that more than one muscle should be examined when evaluating the effects on ostrich meat of extrinsic factors such as diet on fresh meat (Poławska et al., 2013; Dalle Zotte et al., 2014), of additives in processed products (Hoffman et al., 2014) and of storage (Leygonie et al., 2012a).

Supplementation of poultry diet with n-3 enriched sources may increase its susceptibility to oxidation. Thus, the benefit of this composition requires storage conditions that ensure limited lipid oxidation during storage (Filgueras et al., 2011). Freezing is a good method of preservation for meat for relatively long periods (Leygonie et al., 2012a). However, frozen meat storage can affect the structural and chemical 
properties of muscle foods and influence meat quality (Farouk \& Swan, 1998; Leygonie et al., 2012b). Some authors have shown that the fatty acid composition of meat could be modified during storage by oxidative and hydrolytic processes (Alvarez et al., 2009; Diaz et al., 2011), especially that of meat enriched with PUFAs. However, in the current literature there is a shortage of information about the effect of frozen storage time on the fatty acid profiles of ostrich meat. Thus, the aim of this study was to determine the changes in fatty acid profile with special reference to n-3 of ostrich meat supplemented with linseed and rapeseed as related to the frozen storage duration.

\section{Materials and Methods}

Forty ostriches (Struthio camelus var. domesticus) were raised at a commercial farm in Stypułów, western Poland, in 2012/2013. (The farm is under the scientific supervision of the Institute of Genetics and Animal Breeding of the Polish Academy of Sciences.) The birds were raised in a feedlot system with free access to a barn in bad weather. The sides of the pens were made of poles and allowed for interaction between the birds from the various treatments. Ethical clearance was obtained from the local ethical commission (no 27/2009).

Ostriches were raised together in a feedlot system on a commercial ostrich starter diet $(215 \mathrm{~g}$ crude protein $/ \mathrm{kg}$ and $11.92 \mathrm{MJ} / \mathrm{kg}$ feed until 5 months old. From the age of 5 months (ca. $40 \mathrm{~kg} \mathrm{BW}$ ), birds were randomly allocated to five dietary groups: C (control) group, standard diet; L4 group, standard diet supplemented with 4\% linseed; L8 group, standard diet supplemented with $8 \%$ linseed; R5 group, standard diet supplemented with 5\% rapeseed; and R10 group, standard diet supplemented with 10\% rapeseed. The standard diet was based on barley, wheat, wheat bran and soybean meal (Poławska et al., 2012). All diets contained $150 \mathrm{~g}$ crude protein $/ \mathrm{kg}$ and $10.67 \mathrm{MJ} / \mathrm{kg}$ feed. Different dietary fatty acid profiles were achieved by including linseed or rapeseed at various concentrations in the diets. Compared with the control diet, all experimental diets had lower concentrations of SFA (16.1 - 19.9 vs. $21.7 \%$ FA $_{\text {total }}$ ), higher PUFA/SFA (2.6 3.6 vs. 2.2) and lower n-6/n-3 (1.2 - 5.6 vs. 6.3) ratios (Poławska et al., 2012; 2013).

At 12 months old, when the ostriches' live weight reached $96.3 \pm 5.5 \mathrm{~kg}$, they were slaughtered in an EU-approved commercial abattoir for cattle and pigs in Wolbrom (Poland). The ostriches were fasted for $24 \mathrm{~h}$ before being electrically stunned. Bleeding and evisceration were performed according to standard slaughtering procedures for ostriches in Poland (Majewska et al., 2009). Meat samples were taken from the gastrocnemius pars interna (GN) and iliofibularis (IF) muscles from the left side of the carcasses, and transported to the laboratory in insulated containers, where they were maintained at $-20{ }^{\circ} \mathrm{C}$ until further analyses.

Meat samples were cut into steaks (100 - $120 \mathrm{~g}$ each) and vacuum-packed in foil bags (VAC10DT+GV, Edesa Hostelera S.A., Barcelona). The packs were stored in a freezer at $-20^{\circ} \mathrm{C}$ for the duration of the experiment. Samples were analysed at $24 \mathrm{~h}$ after slaughter, and after 60 and 120 days of storage.

Fatty acids were extracted from homogenised samples $(5 \mathrm{~g})$ of ostrich muscles with the chloroformmethanol procedure of Folch et al. (1957). After filtration through filter paper (Filtrak 390), $800 \mu \mathrm{L}$ filtrate was collected into the vials which were then evaporated under nitrogen in a heating block at $50^{\circ} \mathrm{C}$. Samples were saponified with $0.5 \mathrm{M} \mathrm{KOH}$ in methanol into the heating block $\left(75^{\circ} \mathrm{C}\right)$. After saponification, samples were esterified with $4 \%$ solution of $\mathrm{SOCl}_{2}$ in methanol, then the methyl esters were extracted with heptane and salted out with $\mathrm{NaCl}$ to separate the organic layer. Thereafter, $300 \mu \mathrm{L}$ of esters were transferred to vials and $600 \mu \mathrm{L}$ of heptane was added.

Fatty acid methyl esters (FAME) were analysed using a GC-7890 Agilent gas chromatograph equipped with a $60 \mathrm{~m}$ Hewlett-Packard-88 capillary column (Agilent J\&W GC Columns, USA) with $0.25 \mathrm{~mm}$ inner diameter and $0.20 \mu \mathrm{m}$ film thickness. Each sample $(1 \mu \mathrm{L})$ was injected at a split ratio of $1: 40$. Helium was used as a carrier gas at a flow rate of $50 \mathrm{~mL} / \mathrm{min}$. The injector and detector were maintained at $260^{\circ} \mathrm{C}$. Column oven temperature was programmed to increase from $140^{\circ} \mathrm{C}$ (held for $5 \mathrm{~min}$.) at a rate of $4^{\circ} \mathrm{C} / \mathrm{min}$. to $190^{\circ} \mathrm{C}$ and then to $215^{\circ} \mathrm{C}$ at a rate $0.8^{\circ} \mathrm{C} / \mathrm{min}$.

Individual fatty acids were identified by comparing retention times with those of a standard FAME mixture (Supelco 37 Component FAME Mix, 47885-U-10 mg/mL in methylene chloride, analytical standard, Sigma-Aldrich Co.) and expressed as a percentage of total fatty acids.

An analysis of variance using Statistica (version 9, StatSoft Inc., USA) was conducted with diet and storage time as factors. Significant differences $(P<0.05)$ between the means were determined by Tukey's test.

\section{Results and Discussion}

Means for the fatty acid composition in the two muscles as related to duration of frozen storage (up to 120 days) and the types of diet are shown in Tables $1 a, b$ and $2 a, b$. 
Table 1a Saturated and monounsaturated fatty acid composition (\% of fatty acids total) of ostrich gastrocnemius pars interna muscle enriched with two levels of rapeseed and linseed as related to frozen storage time

\begin{tabular}{|c|c|c|c|c|c|c|c|c|c|}
\hline \multirow{2}{*}{ Fatty acids ${ }^{1}$} & \multirow[b]{2}{*}{$\begin{array}{l}\text { Storage } \\
\text { time (S) }\end{array}$} & \multicolumn{7}{|c|}{$\operatorname{Diet}^{3}(D)$} & \multirow{2}{*}{$\frac{\text { Interaction }}{\mathrm{D} \times \mathrm{S}}$} \\
\hline & & C & R5 & R10 & L4 & L8 & Mean & SEM & \\
\hline \multirow[t]{4}{*}{ SFA } & $24 \mathrm{~h}$ & 35.43 & 36.17 & 35.79 & 37.17 & 38.58 & 36.63 & & \\
\hline & $60 \mathrm{~d}$ & 36.40 & 37.23 & 36.75 & 39.88 & 39.78 & 38.01 & & \\
\hline & $120 d$ & 36.65 & 38.40 & 37.52 & 40.89 & 40.24 & 38.74 & & \\
\hline & Mean & 36.16 & 37.26 & 36.68 & 38.57 & 39.53 & & 0.57 & NS \\
\hline \multirow[t]{4}{*}{ 16:0 } & $24 \mathrm{~h}$ & 16.76 & 15.93 & 14.29 & 13.84 & 13.54 & 14.87 & & \\
\hline & $60 \mathrm{~d}$ & 16.91 & 16.06 & 17.12 & 16.15 & 16.90 & 16.63 & & \\
\hline & $120 \mathrm{~d}$ & 17.58 & 16.97 & 17.91 & 16.09 & 16.92 & 17.09 & & \\
\hline & Mean & 16.99 & 16.15 & 16.89 & 15.36 & 15.08 & & 0.12 & NS \\
\hline \multirow[t]{4}{*}{ 18:0 } & $24 \mathrm{~h}$ & 11.04 & 11.18 & 11.19 & 11.42 & 11.83 & 11.33 & & \\
\hline & $60 \mathrm{~d}$ & 10.74 & 10.30 & 10.37 & 11.26 & 11.20 & 10.77 & & \\
\hline & $120 \mathrm{~d}$ & 11.09 & 10.65 & 11.69 & 12.04 & 11.51 & 11.40 & & \\
\hline & Mean & 10.86 & 10.89 & 10.93 & 11.67 & 11.53 & & 0.10 & NS \\
\hline \multirow[t]{4}{*}{ MUFA } & $24 \mathrm{~h}$ & 37.28 & 36.82 & 37.05 & 31.76 & 31.27 & 34.84 & & \\
\hline & $60 d$ & 36.75 & 35.17 & 35.79 & 30.49 & 30.04 & 33.65 & & \\
\hline & $120 \mathrm{~d}$ & 36.48 & 35.18 & 35.04 & 30.39 & 30.36 & 33.49 & & \\
\hline & Mean & $36.84^{\mathrm{a}}$ & $35.72^{\mathrm{a}}$ & $35.95^{a}$ & $30.67^{b}$ & $30.55^{b}$ & & 0.38 & NS \\
\hline \multirow[t]{4}{*}{ 16:1 } & $24 \mathrm{~h}$ & 4.45 & 4.62 & 4.78 & 4.73 & 4.42 & 4.60 & & \\
\hline & $60 \mathrm{~d}$ & 5.89 & 5.75 & 5.70 & 5.04 & 5.20 & 5.52 & & \\
\hline & $120 d$ & 5.90 & 5.57 & 5.95 & 5.27 & 5.54 & 5.65 & & \\
\hline & Mean & $5.41^{\mathrm{a}}$ & $5.08^{\mathrm{ab}}$ & $5.15^{\mathrm{ab}}$ & $4.99^{b}$ & $5.20^{\mathrm{ab}}$ & & 0.07 & NS \\
\hline \multirow[t]{4}{*}{$18: 1 n-9$} & $24 \mathrm{~h}$ & 24.76 & 25.17 & 25.18 & 22.99 & 21.72 & 23.96 & & \\
\hline & $60 \mathrm{~d}$ & 25.15 & 25.46 & 25.94 & 22.80 & 22.36 & 24.34 & & \\
\hline & $120 d$ & 25.32 & 25.60 & 24.08 & 22.52 & 22.12 & 23.93 & & \\
\hline & Mean & $25.01^{\mathrm{a}}$ & $25.38^{a}$ & $25.10^{\mathrm{a}}$ & $22.76^{\mathrm{b}}$ & $22.01^{b}$ & & 0.17 & NS \\
\hline
\end{tabular}

${ }^{1}$ SFA: sum of saturated fatty acids; MFA: sum of monounsaturated fatty acid.

${ }^{2}$ C: control group; L4: diet with $4 \%$ of linseed supplementation; L8: diet with $8 \%$ of linseed supplementation;

R5: diet with $5 \%$ of rapeseed supplementation; R10: diet with $10 \%$ of rapeseed supplementation.

A-C means within rows/columns with different letters differ at $P<0.001$.

${ }^{\mathrm{a}, \mathrm{b}}$ means within rows/columns with different letters differ at $P<0.05$.

${ }^{\mathrm{v}-\mathrm{z}}$ values within rows and columns (significant interaction) with different letters differ at $P<0.05$. 
Table 1b Polyunsaturated fatty acid composition (\% of fatty acids total) of ostrich gastrocnemius pars interna muscle enriched with two levels of rapeseed and linseed as related to frozen storage time

\begin{tabular}{|c|c|c|c|c|c|c|c|c|c|}
\hline \multirow{2}{*}{ Fatty acids ${ }^{1}$} & \multirow[b]{2}{*}{$\begin{array}{l}\text { Storage } \\
\text { time (S) }\end{array}$} & \multicolumn{7}{|c|}{$\operatorname{Diet}^{2}$ (D) } & \multirow{2}{*}{$\frac{\text { Interaction }}{\mathrm{D} \times \mathrm{S}}$} \\
\hline & & C & R5 & R10 & L4 & L8 & Mean & SEM & \\
\hline \multirow[t]{4}{*}{ PUFA } & $24 \mathrm{~h}$ & $27.29^{x y}$ & $27.01^{x y}$ & $27.16^{x y}$ & $31.07^{v}$ & $30.15^{v x}$ & 28.54 & & \\
\hline & $60 d$ & $26.33^{y}$ & $25.79^{z}$ & $27.15^{x y}$ & $30.70^{v x}$ & $30.13^{v x}$ & 28.02 & & \\
\hline & $120 \mathrm{~d}$ & $26.26^{y}$ & $25.77^{z}$ & $26.46^{y}$ & $28.02^{x}$ & $28.40^{x}$ & 26.98 & & \\
\hline & Mean & 26.63 & 26.19 & 26.92 & 29.93 & 29.78 & & 0.27 & 0.03 \\
\hline \multirow[t]{4}{*}{$18: 2 n-6$} & $24 \mathrm{~h}$ & 16.24 & 15.92 & 16.13 & 16.81 & 15.96 & 16.21 & & \\
\hline & $60 d$ & 17.18 & 15.51 & 17.46 & 17.60 & 17.47 & 17.04 & & \\
\hline & $120 d$ & 16.46 & 16.15 & 17.14 & 17.27 & 17.07 & 16.82 & & \\
\hline & Mean & 16.56 & 15.99 & 16.89 & 17.10 & 16.89 & 16.69 & 0.15 & NS \\
\hline \multirow[t]{4}{*}{$18: 3 n-3$} & $24 \mathrm{~h}$ & 1.71 & 1.76 & 1.84 & 5.77 & 4.29 & 3.07 & & \\
\hline & $60 \mathrm{~d}$ & 1.68 & 1.80 & 1.79 & 5.33 & 4.18 & 2.96 & & \\
\hline & $120 d$ & 1.61 & 1.51 & 1.64 & 4.35 & 4.09 & 2.64 & & \\
\hline & Mean & $1.67^{\mathrm{B}}$ & $1.70^{\mathrm{B}}$ & $1.76^{\mathrm{B}}$ & $5.15^{\mathrm{A}}$ & $4.19^{A}$ & & 0.03 & NS \\
\hline \multirow[t]{4}{*}{$20: 4 n-6$} & $24 \mathrm{~h}$ & 8.78 & 8.59 & 8.54 & 7.04 & 8.38 & 8.27 & & \\
\hline & $60 d$ & 7.40 & 7.73 & 7.31 & 6.65 & 7.38 & 7.29 & & \\
\hline & $120 \mathrm{~d}$ & 7.31 & 7.39 & 7.15 & 5.48 & 6.39 & 6.74 & & \\
\hline & Mean & $7.83^{\mathrm{a}}$ & $7.89^{\mathrm{a}}$ & $7.66^{\mathrm{ab}}$ & $6.39^{b}$ & $7.38^{\mathrm{ab}}$ & & 0.41 & NS \\
\hline \multirow[t]{4}{*}{$20: 5 n-3$} & $24 \mathrm{~h}$ & 0.18 & 0.27 & 0.31 & 0.49 & 0.45 & 0.34 & & \\
\hline & $60 d$ & 0.17 & 0.33 & 0.30 & 0.48 & 0.43 & 0.34 & & \\
\hline & $120 d$ & 0.19 & 0.37 & 0.31 & 0.48 & 0.41 & 0.35 & & \\
\hline & Mean & $0.18^{C}$ & $0.34^{\mathrm{B}}$ & $0.30^{\mathrm{B}}$ & $0.48^{\mathrm{A}}$ & $0.43^{\mathrm{A}}$ & & 0.01 & NS \\
\hline \multirow[t]{4}{*}{$22: 6 n-3$} & $24 \mathrm{~h}$ & 0.38 & 0.47 & 0.34 & 0.96 & 1.07 & $0.64^{\mathrm{a}}$ & & \\
\hline & $60 \mathrm{~d}$ & 0.29 & 0.42 & 0.29 & 0.74 & 0.77 & $0.50^{\mathrm{ab}}$ & & \\
\hline & $120 \mathrm{~d}$ & 0.25 & 0.35 & 0.22 & 0.64 & 0.63 & $0.42^{\mathrm{b}}$ & & \\
\hline & Mean & $0.31^{\mathrm{B}}$ & $0.41^{\mathrm{B}}$ & $0.28^{\mathrm{B}}$ & $0.78^{\mathrm{A}}$ & $0.82^{\mathrm{A}}$ & & 0.01 & NS \\
\hline \multirow[t]{4}{*}{$n-6 / n-3$} & $24 \mathrm{~h}$ & $11.02^{v}$ & $9.80^{x}$ & $9.91^{x}$ & $3.30^{z}$ & $4.19^{y z}$ & 7.64 & & \\
\hline & $60 \mathrm{~d}$ & $11.49^{v}$ & $9.11^{x}$ & $10.41^{\mathrm{vx}}$ & $3.70^{z}$ & $4.62^{y}$ & 6.40 & & \\
\hline & $120 \mathrm{~d}$ & $11.60^{v}$ & $10.56^{v x}$ & $11.19^{v}$ & $4.16^{\mathrm{yz}}$ & $4.57^{y}$ & 6.91 & & \\
\hline & Mean & 11.37 & 9.82 & 10.50 & 3.72 & 4.46 & & 0.15 & 0.04 \\
\hline \multirow[t]{4}{*}{ PUFA/SFA } & $24 \mathrm{~h}$ & 0.77 & 0.75 & 0.76 & 0.84 & 0.78 & $0.78^{\mathrm{a}}$ & & \\
\hline & $60 d$ & 0.72 & 0.69 & 0.74 & 0.77 & 0.76 & $0.74^{\mathrm{ab}}$ & & \\
\hline & $120 \mathrm{~d}$ & 0.72 & 0.67 & 0.71 & 0.69 & 0.71 & $0.70^{b}$ & & \\
\hline & Mean & $0.74^{\mathrm{AB}}$ & $0.70^{\mathrm{B}}$ & $0.73^{\mathrm{AB}}$ & $0.76^{\mathrm{A}}$ & $0.75^{\mathrm{A}}$ & & 0.02 & NS \\
\hline
\end{tabular}

\footnotetext{
${ }^{1}$ PUFA: sum of polyunsaturated fatty acids; $n-6 / n-3$ : ratio of $n-6$ to $n-3$ fatty acids; PUFA/SFA: ratio of polyunsaturated to saturated fatty acids.

${ }^{2}$ C: control group; L4 diet with 4\% of linseed supplementation; L8: diet with $8 \%$ of linseed supplementation;

R5: diet with $5 \%$ of rapeseed supplementation; R10: diet with $10 \%$ of rapeseed supplementation.

A-C means within rows/columns with different letters differ at $P<0.001$.

${ }^{\mathrm{a}, \mathrm{b}}$ means within rows/columns with different letters differ at $P<0.05$.

${ }^{\mathrm{v}-\mathrm{z}}$ values within rows and columns (significant interaction) with different letters differ at $P<0.05$.
} 
Table 2a Saturated and monounsaturated fatty acid composition (\% of fatty acid total) of ostrich iliofibularis muscle enriched with two levels of rapeseed and linseed as related to frozen storage time

\begin{tabular}{|c|c|c|c|c|c|c|c|c|c|}
\hline \multirow{2}{*}{$\begin{array}{l}\text { Fatty } \\
\text { acids }^{1}\end{array}$} & \multicolumn{8}{|c|}{$\operatorname{Diet}^{2}$ (D) } & \multirow{2}{*}{$\begin{array}{c}\text { Interaction } \\
\text { D x S }\end{array}$} \\
\hline & $\begin{array}{l}\text { Storage } \\
\text { time (S) }\end{array}$ & C & R5 & R10 & L4 & L8 & Mean & SEM & \\
\hline \multirow[t]{4}{*}{ SFA } & $24 \mathrm{~h}$ & 38.25 & 38.07 & 37.86 & 37.05 & 36.68 & 37.58 & & \\
\hline & $60 \mathrm{~d}$ & 39.36 & 38.54 & 37.84 & 38.85 & 37.30 & 38.37 & & \\
\hline & $120 d$ & 40.18 & 39.26 & 38.91 & 39.99 & 38.42 & 39.35 & & \\
\hline & Mean & 39.26 & 38.62 & 38.20 & 38.63 & 37.13 & & 0.59 & NS \\
\hline \multirow[t]{4}{*}{$16: 0$} & $24 \mathrm{~h}$ & 21.15 & 20.83 & 19.09 & 15.93 & 16.04 & $18.61^{b}$ & & \\
\hline & $60 d$ & 22.82 & 21.28 & 19.86 & 16.63 & 16.94 & $19.51^{\mathrm{a}}$ & & \\
\hline & $120 d$ & 23.31 & 21.77 & 19.79 & 16.86 & 16.92 & $19.73^{a}$ & & \\
\hline & Mean & 22.43 & 21.29 & 19.58 & 16.47 & 16.63 & & 0.16 & NS \\
\hline \multirow[t]{4}{*}{ 18:0 } & $24 \mathrm{~h}$ & 9.64 & 9.51 & 10.02 & 10.44 & 9.80 & 9.88 & & \\
\hline & $60 \mathrm{~d}$ & 9.85 & 9.46 & 9.90 & 10.56 & 10.31 & 10.02 & & \\
\hline & $120 \mathrm{~d}$ & 9.99 & 9.30 & 9.68 & 10.64 & 10.42 & 10.01 & & \\
\hline & Mean & 9.83 & 9.42 & 9.87 & 10.55 & 10.18 & & 0.13 & NS \\
\hline \multirow[t]{4}{*}{ MUFA } & $24 \mathrm{~h}$ & 34.80 & 35.30 & 34.11 & 31.64 & 31.99 & 33.57 & & \\
\hline & $60 \mathrm{~d}$ & 32.70 & 34.15 & 33.20 & 30.53 & 30.22 & 32.16 & & \\
\hline & $120 d$ & 32.89 & 33.58 & 32.28 & 30.76 & 30.06 & 31.91 & & \\
\hline & Mean & $33.45^{\mathrm{a}}$ & $34.34^{\mathrm{a}}$ & $33.20^{\mathrm{a}}$ & $30.98^{b}$ & $30.76^{b}$ & & 0.42 & NS \\
\hline \multirow[t]{4}{*}{$16: 1$} & $24 \mathrm{~h}$ & 6.54 & 6.16 & 5.87 & 4.78 & 4.83 & $5.64^{\mathrm{b}}$ & & \\
\hline & $60 d$ & 6.70 & 6.31 & 5.92 & 5.17 & 5.23 & $5.87^{\mathrm{ab}}$ & & \\
\hline & $120 d$ & 6.99 & 6.61 & 6.10 & 5.42 & 5.37 & $6.10^{\mathrm{a}}$ & & \\
\hline & Mean & $6.74^{\mathrm{a}}$ & $6.36^{\mathrm{a}}$ & $5.96^{\mathrm{a}}$ & $5.12^{\mathrm{b}}$ & $5.14^{b}$ & & 0.10 & NS \\
\hline \multirow[t]{5}{*}{$18: 1 n-9$} & $24 \mathrm{~h}$ & 27.13 & 28.01 & 27.07 & 24.86 & 26.17 & 26.65 & & \\
\hline & $60 \mathrm{~d}$ & 26.88 & 27.99 & 27.15 & 25.21 & 25.85 & 26.61 & & \\
\hline & $120 \mathrm{~d}$ & 26.77 & 27.84 & 27.18 & 25.61 & 25.56 & 26.59 & & \\
\hline & Mean & $26.93^{\mathrm{a}}$ & $27.95^{\mathrm{a}}$ & $27.13^{\mathrm{a}}$ & $25.23^{b}$ & $25.86^{\mathrm{b}}$ & & 0.22 & NS \\
\hline & Mean & $0.67^{\mathrm{B}}$ & $0.68^{\mathrm{B}}$ & $0.73^{\mathrm{AB}}$ & $0.79^{A}$ & $0.81^{A}$ & & 0.04 & NS \\
\hline
\end{tabular}

\footnotetext{
${ }^{1}$ SFA: sum of saturated fatty acids; MUFA: sum of monounsaturated fatty acids.

${ }^{2}$ C: control group; L4: diet with 4\% of linseed supplementation; L8: diet with 8\% of linseed supplementation; R5: diet with $5 \%$ of rapeseed supplementation; R10: diet with $10 \%$ of rapeseed supplementation.

A-B means within rows/columns with different letters differ at $P<0.001$.

${ }^{\mathrm{a}, \mathrm{b}}$ means within rows/columns with different letters differ at $P<0.05$.

${ }^{v-z}$ values within rows and columns (significant interaction) with different letters differ at $P<0.05$.
} 
Table 2b Polyunsaturated fatty acid composition (\% of fatty acid total) of ostrich iliofibularis muscle enriched with two levels of rapeseed and linseed as related to frozen storage time

\begin{tabular}{|c|c|c|c|c|c|c|c|c|c|}
\hline \multirow{2}{*}{$\begin{array}{l}\text { Fatty } \\
\text { acids }^{1}\end{array}$} & \multicolumn{8}{|c|}{$\operatorname{Diet}^{2}$ (D) } & \multirow{2}{*}{$\frac{\text { Interaction }}{D \times S}$} \\
\hline & $\begin{array}{l}\text { Storage } \\
\text { time (S) }\end{array}$ & C & R5 & R10 & L4 & L8 & Mean & SEM & \\
\hline \multirow[t]{4}{*}{ PUFA } & $24 \mathrm{~h}$ & 26.95 & 26.63 & 28.03 & 31.31 & 31.33 & $28.85^{\mathrm{a}}$ & & \\
\hline & $60 \mathrm{~d}$ & 25.93 & 26.31 & 27.96 & 30.62 & 30.49 & $28.26^{\mathrm{ab}}$ & & \\
\hline & $120 d$ & 25.63 & 25.86 & 27.11 & 29.75 & 29.62 & $27.59^{b}$ & & \\
\hline & Mean & $26.17^{b}$ & $26.27^{b}$ & $27.70^{a b}$ & $30.56^{\mathrm{a}}$ & $30.48^{\mathrm{a}}$ & & 0.31 & NS \\
\hline \multirow[t]{4}{*}{$18: 2 n-6$} & $24 \mathrm{~h}$ & 17.25 & 17.16 & 18.92 & 18.09 & 17.90 & 17.86 & & \\
\hline & $60 d$ & 17.71 & 17.59 & 18.30 & 18.11 & 17.69 & 17.88 & & \\
\hline & $120 \mathrm{~d}$ & 17.11 & 17.75 & 18.46 & 18.05 & 18.20 & 17.91 & & \\
\hline & Mean & 17.36 & 17.50 & 18.56 & 18.08 & 17.93 & & 0.12 & NS \\
\hline \multirow[t]{4}{*}{$18: 3 n-3$} & $24 \mathrm{~h}$ & 1.86 & 1.96 & 2.07 & 5.40 & 5.31 & 3.32 & & \\
\hline & $60 \mathrm{~d}$ & 1.79 & 1.85 & 2.00 & 5.21 & 5.20 & 3.21 & & \\
\hline & $120 \mathrm{~d}$ & 1.66 & 1.65 & 1.89 & 4.59 & 4.64 & 2.89 & & \\
\hline & Mean & $1.77^{\mathrm{b}}$ & $1.82^{\mathrm{b}}$ & $1.99^{\mathrm{b}}$ & $5.07^{a}$ & $5.05^{\mathrm{a}}$ & & 0.05 & NS \\
\hline \multirow[t]{4}{*}{$20: 4 n-6$} & $24 \mathrm{~h}$ & 5.77 & 5.60 & 5.99 & 5.21 & 5.65 & $5.64^{\mathrm{a}}$ & & \\
\hline & $60 \mathrm{~d}$ & 5.49 & 5.08 & 5.66 & 4.81 & 5.07 & $5.22^{b}$ & & \\
\hline & $120 d$ & 5.33 & 5.06 & 5.58 & 4.21 & 4.66 & $4.97^{\mathrm{b}}$ & & \\
\hline & Mean & $5.53^{\mathrm{ab}}$ & $5.25^{\mathrm{ab}}$ & $5.74^{\mathrm{a}}$ & $4.74^{\mathrm{b}}$ & $5.13^{\mathrm{ab}}$ & & 0.30 & NS \\
\hline \multirow[t]{4}{*}{$20: 5 n-3$} & $24 \mathrm{~h}$ & 0.49 & 0.43 & 0.50 & 1.30 & 1.20 & $0.78^{\mathrm{a}}$ & & \\
\hline & $60 d$ & 0.47 & 0.42 & 0.50 & 1.10 & 1.09 & $0.72^{\mathrm{ab}}$ & & \\
\hline & $120 d$ & 0.45 & 0.41 & 0.49 & 0.95 & 0.93 & $0.65^{b}$ & & \\
\hline & Mean & $0.47^{\mathrm{B}}$ & $0.42^{\mathrm{B}}$ & $0.50^{\mathrm{B}}$ & $1.12^{\mathrm{A}}$ & $1.07^{\mathrm{A}}$ & & 0.02 & NS \\
\hline \multirow[t]{4}{*}{$22: 6 n-3$} & $24 \mathrm{~h}$ & 0.58 & 0.48 & 0.55 & 1.31 & 1.27 & $0.84^{\mathrm{a}}$ & & \\
\hline & $60 \mathrm{~d}$ & 0.47 & 0.36 & 0.47 & 1.20 & 1.14 & $0.73^{\mathrm{ab}}$ & & \\
\hline & $120 d$ & 0.45 & 0.29 & 0.39 & 0.97 & 0.98 & $0.62^{b}$ & & \\
\hline & Mean & $0.50^{\mathrm{B}}$ & $0.38^{\mathrm{B}}$ & $0.47^{\mathrm{B}}$ & $1.16^{\mathrm{A}}$ & $1.13^{\mathrm{A}}$ & & 0.01 & NS \\
\hline \multirow[t]{4}{*}{$n-6 / n-3$} & $24 \mathrm{~h}$ & $7.86^{x}$ & $7.93^{x}$ & $7.98^{x}$ & $2.91^{y}$ & $3.03^{y}$ & 5.94 & & \\
\hline & $60 d$ & $8.17^{v x}$ & $8.24^{\sqrt{x}}$ & $7.86^{x}$ & $3.01^{y}$ & $3.01^{y}$ & 6.06 & & \\
\hline & $120 d$ & $8.77^{\vee}$ & $9.71^{v}$ & $8.68^{v}$ & $3.42^{y}$ & $3.49^{y}$ & 6.81 & & \\
\hline & Mean & 8.26 & 8.63 & 8.17 & 3.11 & 3.18 & & 0.17 & 0.04 \\
\hline \multirow[t]{4}{*}{ PUFA/SFA } & $24 \mathrm{~h}$ & 0.70 & 0.70 & 0.74 & 0.85 & 0.85 & 0.77 & & \\
\hline & $60 \mathrm{~d}$ & 0.66 & 0.68 & 0.74 & 0.79 & 0.82 & 0.74 & & \\
\hline & $120 d$ & 0.64 & 0.66 & 0.70 & 0.74 & 0.77 & 0.70 & & \\
\hline & Mean & $0.67^{\mathrm{B}}$ & $0.68^{\mathrm{B}}$ & $0.73^{\mathrm{AB}}$ & $0.79^{\mathrm{A}}$ & $0.81^{A}$ & & 0.04 & NS \\
\hline
\end{tabular}

${ }^{1}$ PUFA: sum of polyunsaturated fatty acids; n-6/n-3: ratio of $n-6$ to $n-3$ fatty acids;

PUFA/SFA: ratio of polyunsaturated to saturated fatty acids.

${ }^{2}$ C: control group; L4: diet with $4 \%$ of linseed supplementation; L8: diet with $8 \%$ of linseed supplementation;

R5: diet with $5 \%$ of rapeseed supplementation; R10: diet with $10 \%$ of rapeseed supplementation.

$A-B$ means within rows/columns with different letters differ at $P<0.001$;

${ }^{\mathrm{a}, \mathrm{b}}$ means within rows/columns with different letters differ at $P<0.05$;

${ }^{\mathrm{v}-\mathrm{z}}$ values within rows and columns (significant interaction) with different letters differ at $P<0.05$.

\section{Conclusions}

A decrease was noted in the PUFA concentration in ostrich meat as related to frozen storage duration. The results suggest that for up to 60 days freezing $\left(-20^{\circ} \mathrm{C}\right)$ is an acceptable method of preservation for 
ostrich meat enriched with n-3 fatty acids. However, the changes in PUFA profile in the second period of storage (61 - 120 days) indicate that further research should be conducted to evaluate a prolonged frozen storage for 180 days or longer. It would also be worthwhile to evaluate the effect of prolonged frozen storage of ostrich muscle on other quality parameters such as drip loss, colour stability and sensory attributes. However, it is always a challenge to conduct such studies, especially when one considers sensory analyses, although the use of TBARs as a measurement of lipid oxidation may be more feasible.

\section{Acknowledgments}

This study was financed within the project BIOFOOD (innovative, functional products of animal origin, POIG.01.01.02-014-090/09), which was co-financed by the European Regional Development Fund within the Innovative Economy Operational Programme 2007-2013.

\section{References}

Alvarez, I., De La Fuente, J., Cañeque, V., Lauzurica, S., Pérez, C. \& Dìaz, M.T., 2009. Changes in the fatty acid composition of $m$. longissimus dorsi of lamb during storage in a high-oxygen modified atmosphere at different levels of dietary vitamin E supplementation. J. Agric. Food Chem. 57, 140-146.

Cooper, R.G. \& Horbańczuk, J.O., 2004. Ostrich nutrition: a review from a Zimbabwean perspective. Monography. Revue Scientifique et Technique de I' Office International Des Epizooites. 23 (3), 1033-1042.

Cosgrove, J.P., Church, D.F. \& Pryor, W.A., 1987. The kinetics of the autoxidation of polyunsaturated fatty acids. Lipids 22, 299-304.

Dalle Zotte, A., Brand, T.S., Hoffman, L.C., Schoon, K., Cullere, M. \& Swart, R., 2013. Effect of cottonseed oilcake inclusion on ostrich growth performance and meat chemical composition. Meat Sci. 93, 194-200.

Díaz, M.T., Cañeque, V., Sánchez, C.I., Lauzurica, S., Pérez, C., Fernández, C., Álvarez, I. \& De la Fuente J., 2011. Nutritional and sensory aspects of light lamb meat enriched in n-3 fatty acids during refrigerated storage. Food Chem. 124, 147-155.

Farouk, M.M. \& Swan, J.E., 1998. Effect of rigor temperature and frozen storage on functional properties of hot-boned manufacturing beef. Meat Sci. 49, 233-247.

Filgueras, R.S., Gatellier, P., Zambiazi, R.C. \& Santé-Lhoutellier, V., 2011. Effect of frozen storage duration and cooking on physical and oxidative changes in $m$. Gastrocnemius pars interna and $m$. lliofibularis of Rhea americana. Meat Sci. 88, 645-651.

Folch, J., Lee, M. \& Sloane Stanley, G.H., 1957. A simple method for the isolation and purification of total lipids from animal tissues. J. Biol. Chem. 22, 226-497.

Girolami, A., Marsico, I., D'Andrea, G., Braghieri, A., Napolitano, F., Cifuni, G.F., 2003. Fatty acid profile, cholesterol content and tenderness of ostrich meat as influenced by age at slaughter and muscle type. Meat Sci. 64, 309-315.

Hoffman, L.C., 2008. Value adding and processing of ratite meat: A review. Aust. J. Exp. Agric. 48 (10), $1270-1275$.

Hoffman, L.C., Joubert, M., Brand, T.S. \& Manley, M., 2005. The effect of dietary fish oil rich in n-3 fatty acids on the organoleptic, fatty acid and physicochemical characteristics of ostrich meat. Meat Sci. 70 , 45-53.

Hoffman, L.C., Jones, M., Muller, N.M., Joubert, E. \& Sadie, A., 2014. Lipid and protein stability and sensory evaluation of Ostrich (Struthio camelus) droëwors with the addition of rooibos tea extract (Aspalathus linearis) as a natural antioxidant. Meat Sci. 96, 1289-1296.

Horbańczuk, J., Sales, J., Celeda, T., Konecka, A., Zięba, G. \& Kawka, P., 1998. Cholesterol content and fatty acid composition of ostrich meat as influenced by subspecies. Meat Sci. 50, 385-388.

Horbańczuk, J.O, Tomasik, C. \& Cooper, R.G., 2008. Ostrich farming in Poland - its history and current situation after accession to the European Union. Avian Poult. Biol. Rev. 1, 65-71.

Leygonie, C., Britz, T.J. \& Hoffman, L.C., 2012a. Meat quality comparison between fresh and frozen/thawed ostrich $m$. iliofibularis. Meat Sci. 91, 364-368.

Leygonie, C., Britz, T.J. \& Hoffman, L.C., 2012b. Impact of freezing and thawing on the quality of meat: Review. Meat Sci. 91, 93-98.

Majewska, D., Jakubowska, M., Ligocki, M., Tarasewicz, Z., Szczerbińska, D., Karamucki, T. \& Sales, J., 2009. Physicochemical characteristics, proximate analysis and mineral composition of ostrich meat as influenced by muscle. Food Chem. 117, 207-211. 
Poławska, E., Lisiak, D., Jóźwik, A., Pierzchała, M., Strzałkowska, N., Pomianowski, J. \& Wójcik, A., 2012. The effect of the diet supplementation with linseed and rapeseed on the physico-chemical and sensory characteristics of ostrich meat. Anim. Sci. Pap. Rep. 30, 65-72.

Poławska, E., Horbańczuk, J., Pierzchała, M., Strzałkowska, N., Jóźwik, A., Wójcik, A., Pomianowski, J., Gutkowska, K., Wierzbicka, A. \& Hoffman, L.C., 2013. Effect of dietary linseed and rapeseed supplementation on the fatty acid profiles in the ostrich. Part 1. Muscles. Anim. Sci. Pap. Rep. 31 (3), 239-248.

Sales, J. \& Horbańczuk, J., 1998. Ratite meat. Wrld Poult. Sci. J. 54, 59-67.

Santos-Filho, J.M., Morais, S., Rondina, D., Beserra, F., Neiva, J.N. \& Magalhaes, E.F., 2005. Effect of cashew nut supplemented diet, castration and time of storage on fatty acid composition and cholesterol content of goat meat. Small Rumin. Res. 57, 51-56.

Zymon, M., Strzetelski, J., Pustkowiak, H. \& Sosin, E., 2007. Effect of freezing and frozen storage on fatty acid profile of calves' meat. Polish J. Food Nutr. Sci. 57, 647-650.

World Health Organization/Food and Agriculture Organization (WHO/FAO). (2003). Diet nutrition and the prevention of chronic diseases. WHO, Geneve. pp. 4-101. 